\title{
Mitigating Damage to Hybrid Perovskites Using
}

\section{Pulsed-Beam TEM}

\author{
Elisah J. VandenBussche, Catherine P. Clark, Russell J. Holmes, and David J. Flannigan* \\ Department of Chemical Engineering and Materials Science, University of Minnesota, 421 \\ Washington Avenue SE, Minneapolis, MN 55455, USA
}

\begin{abstract}
Using a pulsed-beam TEM, we discover a reduction in damage to $\mathrm{MAPbI}_{3}$ as compared to conventional beams delivered at the same dose rates. For rates as low as $0.001 \mathrm{e} \AA^{-}$ ${ }^{2} \cdot \mathrm{s}^{-1}$, we find up to a $17 \%$ reduction in damage at a total dose of $10 \mathrm{e} \cdot \AA^{-2}$. We systematically study the effects of number of electrons in each pulse, as well as the duration between pulse arrival. Damage increases for both, though the number of electrons per pulse has a larger effect. A crossover is identified, where a pulsed beam causes more damage than a conventional one. Though qualitatively similar to previous findings, the degree to which damage is reduced in $\mathrm{MAPbI}_{3}$ is less than has been observed for other materials (e.g., $\mathrm{C}_{36} \mathrm{H}_{74}$ ), supporting the hypothesis that the effects are material and damage-mechanism dependent. Despite this, the observation here of damage reduction for relatively large electron packets (up to 200 electrons per pulse) suggests $\mathrm{MAPbI}_{3}$ is in fact less susceptible to irradiation than $\mathrm{C}_{36} \mathrm{H}_{74}$, which may be related to reported self-healing effects. This work provides insights into damage processes and durability in the hybrid perovskites and also illustrates the viability of using pulsed-beam TEM to explore the associated molecular-level routes to degradation, analogous to laser-accelerated energetic pulsed electron beams and the study of damage to biomolecules, cells, and tissues in radiobiology.
\end{abstract}

Keywords: methylammonium lead iodide, radiation damage, electron microscopy, electronphonon coupling, thermal transport 


\section{Introduction}

Comprehensive understanding of high power-conversion efficiencies of hybrid organicinorganic perovskite (HOIP) photovoltaic cells requires elucidation of atomic and nanoscale properties and behaviors of these materials. ${ }^{1,2}$ Structural, chemical, electronic, and dynamic properties at this scale are accessible with transmission electron microscopy (TEM), but the stability of HOIPs is such that electron-beam damage can be significant, while connections to photon-induced damage can be drawn. ${ }^{3-5}$ Indeed, sensitivity of HOIPs to even low dose rates ( 1 $\mathrm{e} \cdot \AA^{-2} \cdot \mathrm{s}^{-1}$ ) may limit what can be learned about fundamental structure/function relationships and illustrates the importance of developing new methods for probing degradation and uncovering new behaviors. ${ }^{6-8}$ For example, damage during electron-beam irradiation is thought to occur through a combination of charging, ionic excitation, and heating leading to ion migration and separation of the organic and inorganic constituents. ${ }^{8,9}$ As such, a number of methods, such as cryo-electron microscopy and low-dose imaging and diffraction, have been used to reduce deleterious beam effects. ${ }^{10,11}$

Femtosecond (fs) laser-driven and picosecond chopped-beam sources in modified TEMs offer additional means for mitigating and studying damage. ${ }^{12-16}$ These methods employ temporally-modulated beams, where electrons are delivered to the specimen in discrete pulses with well-defined durations between each, rather than in the random fashion of conventional sources (e.g., thermionic). Indeed, time-dependent aspects of charging and thermal energy, as well as the dynamic self-healing properties of HOIPs, suggest there may be insights into these temporal mechanisms that can be achieved by using well-timed pulses. ${ }^{3,17-19}$ Accordingly, here we explore fs laser-driven pulsed TEM for studying damage in HOIPs, specifically methylammonium lead iodide $\left(\mathrm{CH}_{3} \mathrm{NH}_{3} \mathrm{PbI}_{3}\right.$ or $\left.\mathrm{MAPbI}_{3}\right)$. Our main goal was to determine - all 
else being equal - if a pulsed beam leads to a reduction in $\mathrm{MAPbI}_{3}$ damage compared to a conventional beam. Accordingly, we focused on quantitatively comparing damage caused by pulsed beams to that of conventional thermionic beams at the same dose rates and the same total doses. We also studied the effects of the number of electrons per pulse (e/p) and the duration between pulse arrival at the specimen ( $f^{-1}$, where $f$ is the laser repetition rate). We find a clear reduction in damage for pulsed beams compared to random (thermionic) beams, as well as an apparent exacerbation of damage with increasing instantaneous dose (i.e., with more electrons per pulse) even with longer time elapsed between the arrival of each. We also observe a crossover effect, where pulsed beams become more detrimental than random beams. Interestingly, the degree to which damage is reduced in $\mathrm{MAPbI}_{3}$ is lower than that for $\mathrm{C}_{36} \mathrm{H}_{74}$, supporting the hypothesis that the pulsed-beam effect is material and mechanism dependent.

\section{Results and Discussion}

Beam-damage mechanisms in TEM are numerous and often synergistic, necessitating detailed design and systematic execution of experiments. ${ }^{5,20}$ Conveniently, fs pulsed lasers in a stable lab environment afford high levels of control, enabling accurate and precise quantification of pulsed TEM beam damage. ${ }^{14}$ An overview of the fs laser-driven approach used here, and the method for quantifying damage to $\mathrm{MAPbI}_{3}$ specimens, is shown in Figure 1. Temporal regularity of the pulsed electron beam was accomplished using a 300-fs pulsed laser (PHAROS, Light Conversion), which confines electron emission to a train of 300-fs windows evenly spaced in time by $f^{1}$. The pulse train was generated via the photoelectric effect in a modified $200 \mathrm{kV} \mathrm{TEM}$ (Tecnai Femto, Thermo Fisher) using ultraviolet laser pulses $(h v=4.8 \mathrm{eV})$ and a $\mathrm{LaB}_{6}$ electron source $(\varphi=2.4 \mathrm{eV}$; Fig. 1a). Dose rate was controlled with both the laser-pulse fluence (which 
dictates e/p) and $f$ (which dictates the number of pulses per second and the specimen relaxation time between each pulse, $f^{1}$ ).
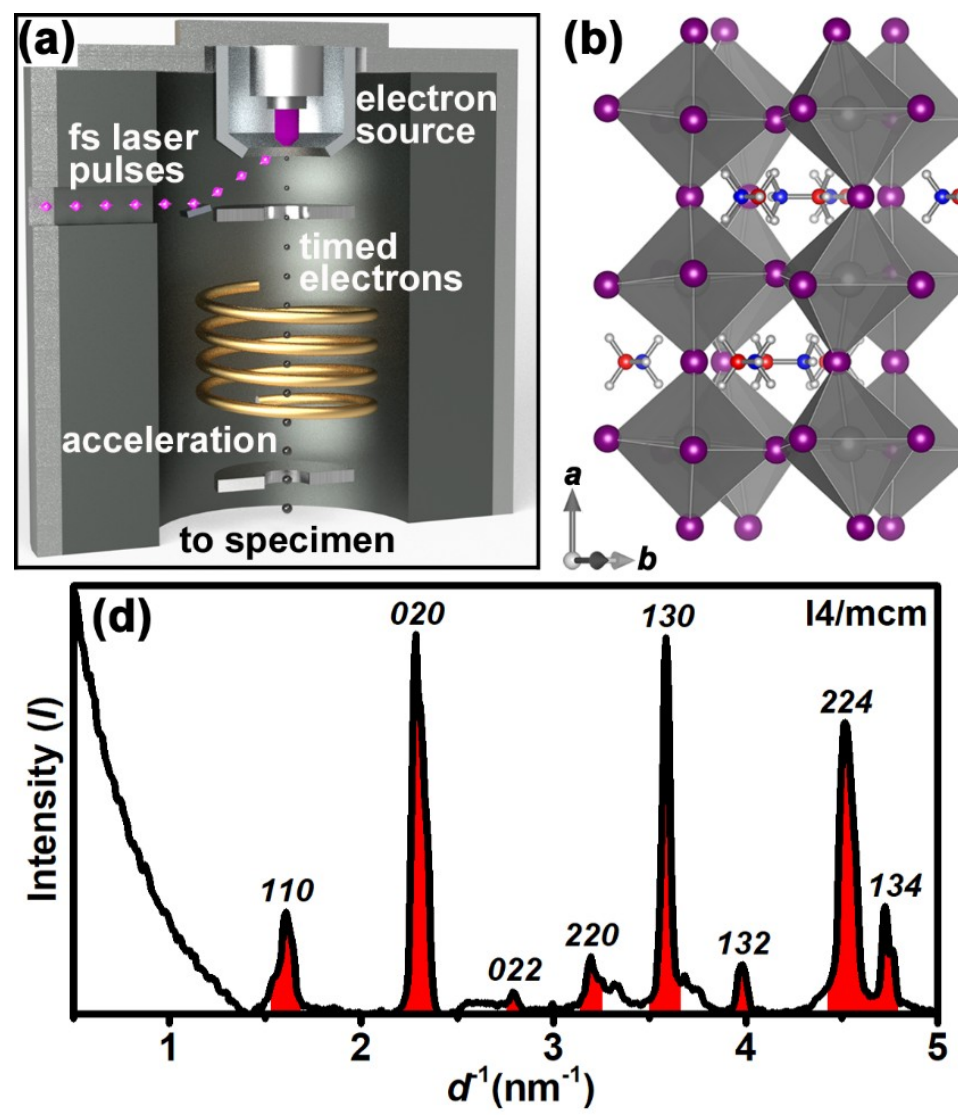
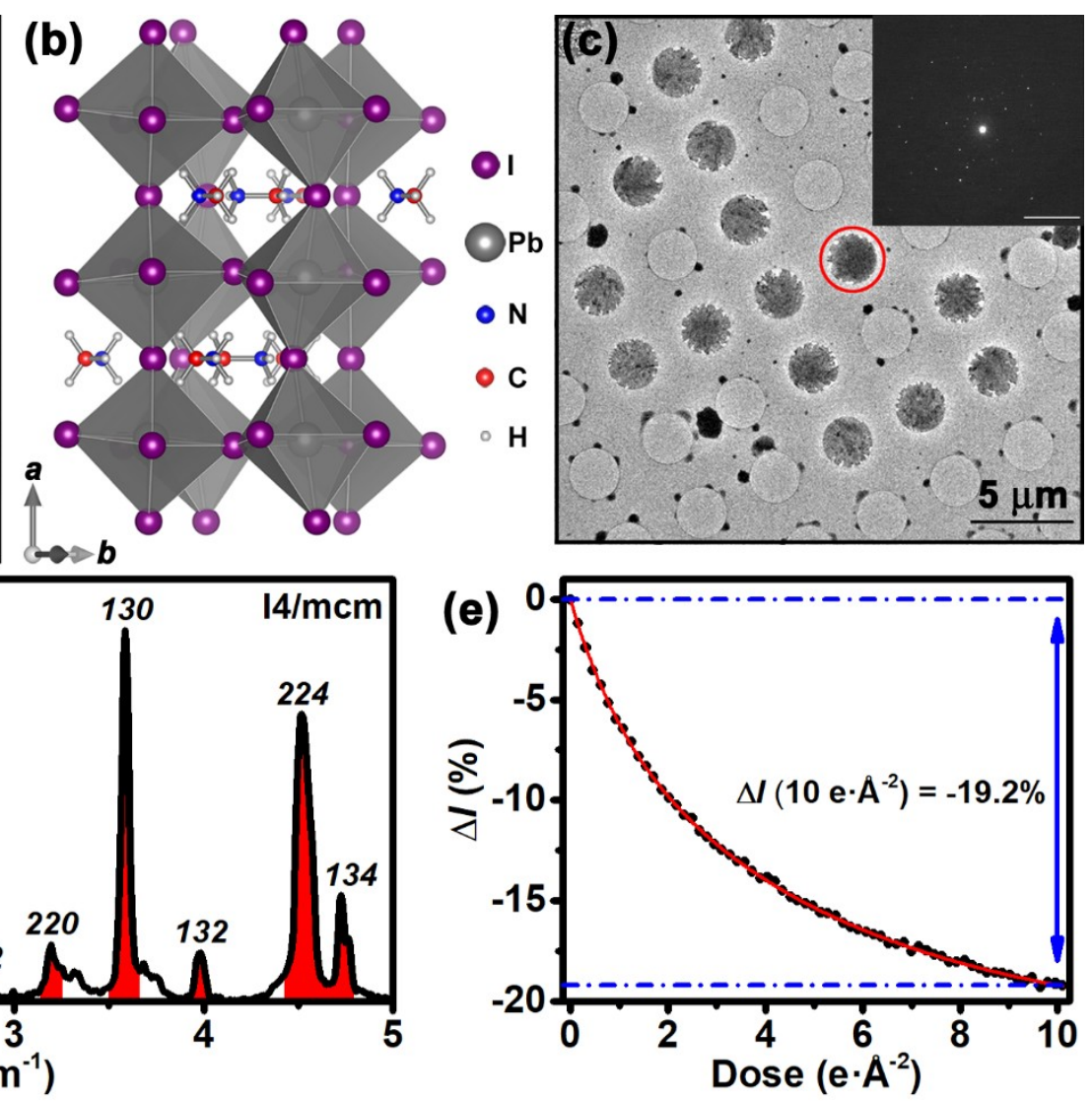

Figure 1. Laser-driven pulsed-beam TEM and quantification of damage to $\mathrm{MAPbI}_{3}$.

Simplified schematic of the electron-source region with pertinent aspects and components labeled. Adapted with permission from VandenBussche, E. J.; Flannigan, D. J. Nano Lett. 2019, 19, 6687-6694. Copyright 2019 American Chemical Society.

(b) Structure of $\mathrm{MAPbI}_{3} \cdot{ }^{21}$

Crystallographic axes are shown in the lower left of the panel. (c) Low-magnification brightfield image of the TEM specimens. The diffraction pattern (inset; scale bar $=5 \mathrm{~nm}^{-1}$ ) was obtained from the red-circled region. (d) Intensity plot from azimuthally averaging the pattern in (c). Red peaks are those used for monitoring beam-induced intensity changes. The space group 
used for indexing was $I 4 / \mathrm{mcm}$, though the structure is not yet universally agreed upon. ${ }^{22,23}$ (e) Bragg-intensity fading curve for a pulsed electron beam (dose rate $=0.001 \mathrm{e} \cdot \AA^{-2} \cdot \mathrm{s}^{-1}$; beam size $=$ $\left.245 \pm 3 \mu \mathrm{m}^{2} ; 50.4 \pm 1.0 \mathrm{e} / \mathrm{p} ; f^{1}=2 \mu \mathrm{s}\right)$. Red curve is to determine $\Delta I$ at $10 \mathrm{e} \cdot \AA^{-2}$, which here is $-19.2 \%$.

Specimens were synthesized by spin coating a $0.3-\mathrm{M}$ solution of $\mathrm{MAPbI}_{3}$ with $10 \%$ molar excess MAI in a 4:1 volume ratio of DMF:DMSO onto holey amorphous-carbon grids (Quantifoil, Electron Microscopy Sciences) (Fig. 1b,c). First, the grid was reversibly adhered to a silicon support using a drop of toluene followed by heating at $100{ }^{\circ} \mathrm{C}$ for $1 \mathrm{~min}$. Next, $0.2 \mathrm{~mL}$ of $\mathrm{MAPbI}_{3}$ solution was dropped onto the supported grid while spinning at $3000 \mathrm{rpm}$ for $2 \mathrm{~min}$. Finally, the grid was annealed at $100{ }^{\circ} \mathrm{C}$ for $1 \mathrm{hr}$. This produced freestanding, polycrystalline $\mathrm{MAPbI}_{3}$ islands spanning the 2.5- $\mu \mathrm{m}$ holes of the grids (Fig. 1c,d). Accordingly, each island was determined from the bright-field TEM images to be $4.8 \pm 0.2 \mu \mathrm{m}^{2}$ in area (error is one standard deviation from the mean). Damage was quantified using a fading-curve method, where a reduction in Bragg-beam intensities ( $\Delta I$; indicative of destruction of $\mathrm{MAPbI}_{3}$ crystalline order) was tracked as a function of accumulated dose (Fig. 1e). ${ }^{24}$ Peaks arising from planes with $d<6.3$ $\AA$ were used to monitor damage effects for both beam types (i.e., pulsed and conventional). Note that $\Delta I=\left(I-I_{0}\right) / I_{0}$, which is the normalized change in intensity relative to that at nominally zero dose $\left(I_{0}\right)$. A total accumulated dose of $10 \mathrm{e} \cdot \AA^{-2}$ was used as the reference point throughout.

Comparison of damage caused to $\mathrm{MAPbI}_{3}$ by a pulsed beam to that of a conventional thermionic beam is shown in Figure 2. For a common dose rate $\left(0.001 \mathrm{e} \cdot \AA^{-2} \cdot \mathrm{s}^{-1}\right.$ for an illuminated area of $245 \pm 3 \mu \mathrm{m}^{2}$ ), there is a clear reduction in the extent to which the intensities fade for the pulsed beam under the conditions used for this experiment $\left(50.4 \pm 1.0 \mathrm{e} / \mathrm{p}\right.$ and $f^{-1}=2$ 
$\mu \mathrm{s})$. At a total accumulated dose of $10 \mathrm{e} \cdot \AA^{-2}$, the intensity change for the pulsed beam is $-19.2 \%$, while that of the thermionic beam is $-23.2 \%$. This shows that providing temporally-regular pauses in electron-beam irradiation, and thus providing regular periods of specimen relaxation and a reduction in exacerbating effects (e.g., multi-electron impact within a specific damage radius), leads to enhanced preservation of $\mathrm{MAPbI}_{3}$ structural order compared to conventional random-beam low-dose methods. Note that for pulsed-beam TEM experiments performed on $\mathrm{C}_{36} \mathrm{H}_{74}$ and bacteriorhodopsin, the extent to which damage was reduced was greater than seen here, though evidence indicates the degree of reduction also depends on $f^{1} .^{14,16}$ This suggests the extent to which damage is reduced is material and mechanism dependent (perhaps strongly so) and that the overall effect of pulsed beams with respect to damage reduction is a general one.

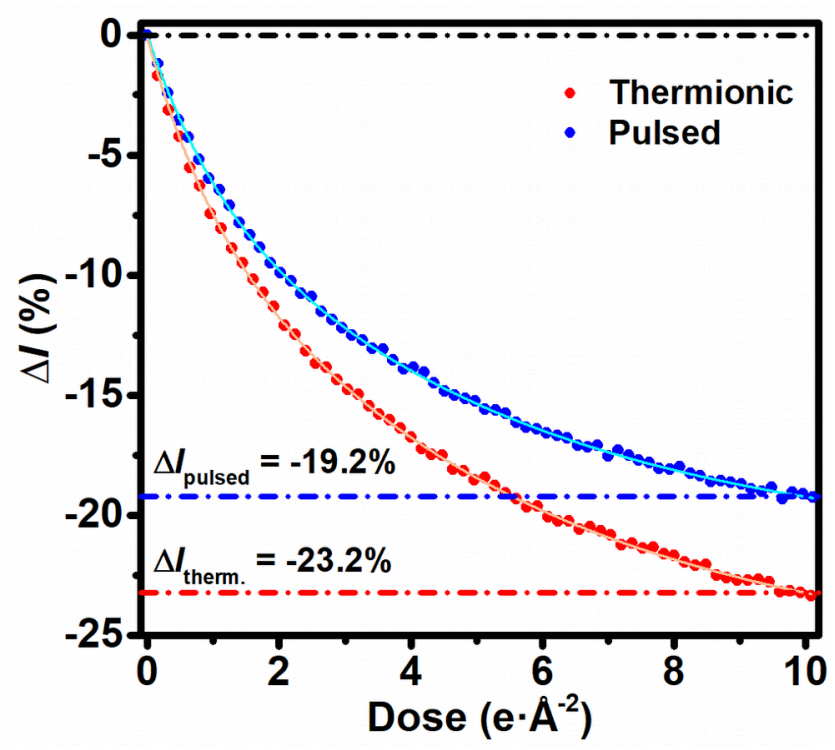

Figure 2. Bragg-peak intensity fading curves for $\mathrm{MAPbI}_{3}$ for pulsed (blue; also shown in Fig. 1e) and thermionic (red) beams delivered at the same dose rate $\left(0.001 \mathrm{e} \cdot \AA^{-2} \cdot \mathrm{s}^{-1}\right)$. Fits to the data are for determining $\Delta I$ at $10 \mathrm{e} \cdot \AA^{-2}$, which here is $-19.2 \%$ for the pulsed beam and $-23.2 \%$ for the conventional thermionic beam (as indicated by the blue and red horizontal dot-dashed lines, 
respectively). The beam was spread over an area of $245 \pm 3 \mu \mathrm{m}^{2}$ for both types. The pulsed beam consisted of $50.4 \pm 1.0 \mathrm{e} / \mathrm{p}$ and $f^{1}=2 \mu$ s (i.e., a $500-\mathrm{kHz}$ repetition rate).

A number of effects and conditions - in addition to beam-induced damage to crystalline order - can produce an apparent dose-dependent drop in Bragg-beam intensities. Thus, drawing conclusions from direct comparisons made across multiple measurements and specimens requires control of myriad variables that might influence, interfere with, and overwhelm the intrinsic beam-damage behavior (e.g., specimen thickness and lateral dimensions, specimen bending under the beam, lab and instrument temperature stability, specimen and beam drift, etc.). Accordingly, sources of error and artifacts were identified and accounted for via control experiments and monitoring of experimental conditions. Detailed descriptions of the measures taken here are reported elsewhere. ${ }^{14}$ As an example of the requirements needing to be met for data to be accepted, experiments were rejected and repeated when the beam current, as well as the beam size, differed by more than $1 \%$ before and after acquisition of a data series. Experiments were conducted after observable directional specimen drift ceased. Further, experiments were rejected if the specimen was found to have directionally drifted more than $1 \%$ of the substrate hole diameter (i.e., $25 \mathrm{~nm}$ ) between the start and the finish of data acquisition. Pre-irradiation was eliminated by navigating specimen movement systematically and ensuring no two experiments were conducted within two beam diameters of one another. Data presented here consists of multiple experimental trials conducted over several days.

To better understand the origins of damage reduction when using a pulsed beam, effects of varying e/p and $f^{1}$ were systematically explored (Fig. 3). Depending upon the mechanisms at work, one might intuitively expect an increase in damage with increased e/p, due to more 
inelastic electron/specimen collisions occurring within a given pulse, and also with decreased $f^{1}$, due to there being less time between electron arrival at the specimen (thus reducing relaxation and recovery time and increasing the probability of simultaneous or near-simultaneous impact within a particular damage radius). ${ }^{14-16}$ However, regimes do exist wherein the benefits gained by increasing $f^{1}$ are lost when simultaneously increasing e/p, as observed for $\mathrm{C}_{36} \mathrm{H}_{74}{ }^{14}$ This is indeed the case for $\mathrm{MAPbI}_{3}$. For dose rates of $0.001 \mathrm{e} \cdot \AA^{-2} \cdot \mathrm{s}^{-1}$ and $0.01 \mathrm{e} \cdot \AA^{-2} \cdot \mathrm{s}^{-1}$ (Fig. 3a and 3b, respectively), damage increases with increased e/p despite an accompanying modest increase in $f$ ${ }^{1}$ from $2 \mu$ s to $4 \mu$ s for the larger electron pulses. Such an effect was also observed in single crystals of $\mathrm{C}_{36} \mathrm{H}_{74}$, where going from $1 \mathrm{e} / \mathrm{p}$ to $5 \mathrm{e} / \mathrm{p}$ had a substantially larger effect on damage than did decreasing $f^{1}$ from $20 \mu$ s to $5 \mu$ s. ${ }^{14}$ Taken altogether, these results suggest that specimen relaxation and recovery processes that are at work during the few microseconds between pulses can be overwhelmed by exacerbating effects of multi-electron impact within a given damage radius. That is, additional energy deposited into the already-excited specimen region causes further structural damage that otherwise would have recovered - or would have been exponentially less significant (owing to exponential relationships for reaction kinetics, diffusion rates, and thermal energy) - during a single electron event isolated in space and time. 

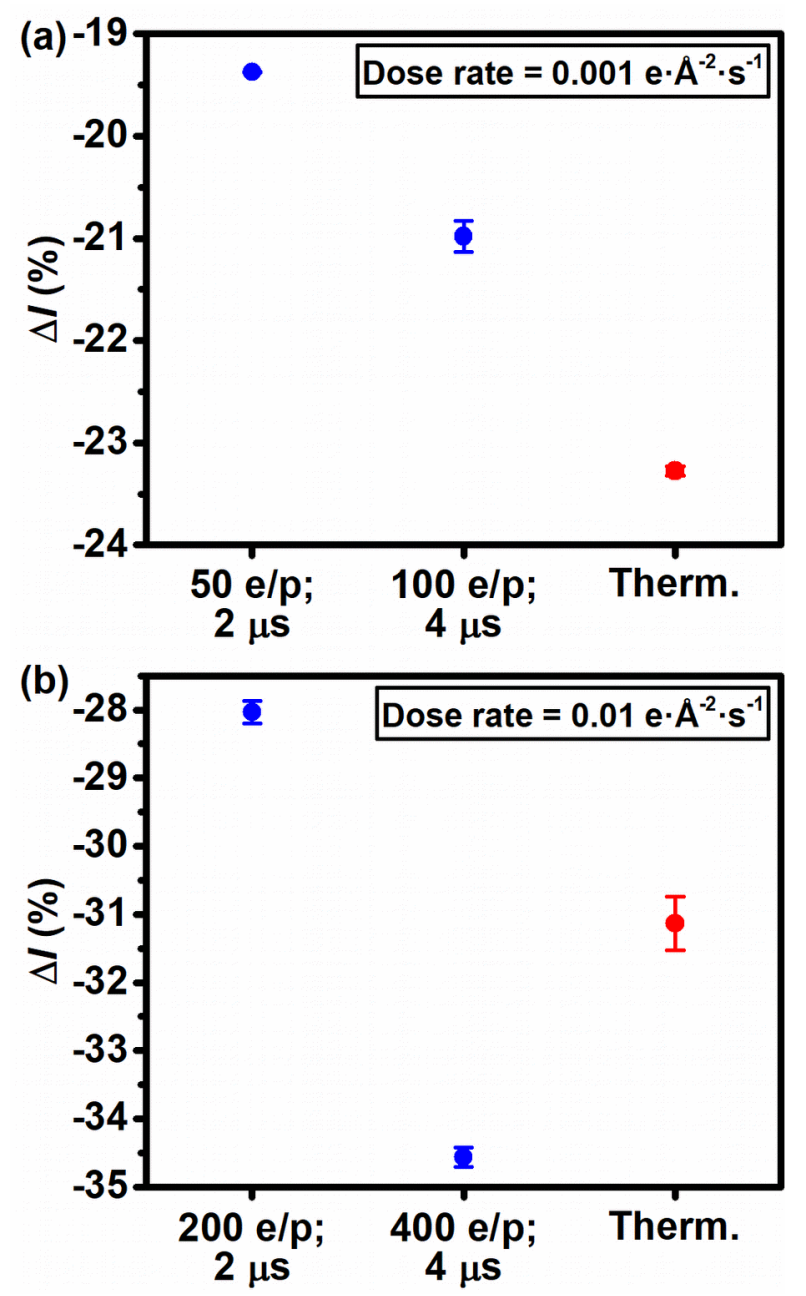

Figure 3. Effect of e/p and $f^{1}$ on damage to $\mathrm{MAPbI}_{3}$ for a total dose of $10 \mathrm{e} \cdot \AA^{-2}$. (a) Braggintensity reduction $(\Delta I)$ for pulsed (blue) and thermionic (therm., red) beams administered at a dose rate of $0.001 \mathrm{e} \cdot \AA^{-2} \cdot \mathrm{s}^{-1}$. The time between electron pulses in microseconds $\left(f^{-1}\right)$ is noted below the number of electrons per pulse (e/p). Error bars for $50 \mathrm{e} / \mathrm{p}, 100 \mathrm{e} / \mathrm{p}$, and thermionic are one standard deviation over 2, 4, and 2 separate experiments, respectively. (b) Bragg-intensity reduction $(\Delta I)$ for pulsed (blue) and thermionic (therm., red) beams administered at a dose rate of $0.01 \mathrm{e} \cdot \AA^{-2} \cdot \mathrm{s}^{-1}$. Error bars for $200 \mathrm{e} / \mathrm{p}, 400 \mathrm{e} / \mathrm{p}$, and thermionic are one standard deviation over 2, 2, and 3 separate experiments, respectively. 
In addition to the overall trends shown in Figure 3, a type of crossover or threshold region exists, where more - rather than less - damage is caused by the pulsed beam as compared to a conventional beam delivered at the same dose rate (Fig. 3b). Here, such a crossover is seen when increasing from 200 e/p separated by $2 \mu$ s to 400 e/p separated by $4 \mu$ s. This behavior lends support to the hypothesis that it is multi-electron impact leading to compounding effects that is driving the dramatic increase in damage with increasing e/p. ${ }^{14}$ In essence, this is due to additional energy being deposited into already-excited specimen regions prior to full relaxation back to ground-state conditions. Interestingly, the instantaneous dose rate for a single pulse of 400 electrons confined to a 300-fs window (more likely a few picoseconds due to Coulombic expansion $)^{25}$ is $5 \times 10^{4} \mathrm{e} \cdot \AA^{-2} \cdot \mathrm{s}^{-1}$, assuming uniform illumination across the $245-\mu \mathrm{m}^{2}$ beam area. It is therefore perhaps surprising that such a beam does not produce substantially more damage than observed, though the number of electrons per $\AA^{2}$ per pulse is only $2 \times 10^{-8}$. Though the time-averaged reduction in damage for pulsed beams is clear, pulse-to-pulse behaviors and the resulting specimen effects are largely unknown and require additional investigation.

Damage reduction (and increase at the crossover) to $\mathrm{MAPbI}_{3}$ when using pulsed-beam TEM is likely driven by temporal processes that are active on the pulse-to-pulse timescales. ${ }^{12-16}$ Such processes include thermal effects which, while not yet well understood for electron-beam excitation and damage in $\mathrm{MAPbI}_{3}$, are important in similar low-thermal-conductivity materials..$^{20,24,26}$ The effect of thermal processes in damage reduction can be appreciated by noting timescales of thermal diffusion and relaxation in $\mathrm{MAPbI}_{3}$ relative to $f^{1}$. Using known constants, ${ }^{27-30}$ it is estimated that thermal energy deposited into a 2.5- $\mu$ m diameter $\mathrm{MAPbI}_{3}$ crystal would largely dissipate into the carbon substrate within several microseconds. Note, however, that temperature from the perspective of the entire specimen is likely too coarse a view 
when considering pulsed-beam damage mechanisms and temporal aspects of molecular-scale excitations. Nevertheless, this estimate indicates thermal-dissipation times are comparable to $f^{1}$, suggesting complete relaxation of increased vibrational energies prior to a subsequent inelastic collision is a plausible source of reduced damage when electron delivery to the specimen is strictly temporally controlled.

Timescales of electron-phonon coupling and lattice thermalization are also worth noting. Electron-phonon coupling in $\mathrm{MAPbI}_{3}$ films occurs on the order of hundreds of femtoseconds, while lattice thermalization takes a few picoseconds. ${ }^{31}$ Owing to the electron-pulse duration, most or all will arrive within this time frame. Further, increasing e/p generally causes an increase in pulse duration, ${ }^{25}$ thus creating an environment where the likelihood of exacerbating effects contributing to damage is increased. Systematic pulse duration experiments may shed light on such effects. Indeed, similar arguments can be made regarding specimen charging, charge dissipation, and electron-pulse durations and timing.

\section{Conclusions}

In conclusion, for common total doses and dose rates, we have discovered a regime where using a pulsed-beam TEM leads to reduced damage to $\mathrm{MAPbI}_{3}$. The degree of reduction is enhanced by using pulses with fewer electrons, while the duration between pulses appears to have a smaller but still non-trivial effect. We also discover a crossover effect, in which pulsed beams cause more damage than an otherwise identical conventional beam. This constitutes the first example of these effects for direct-comparison experiments at dose rates commonly used in low-dose TEM. The findings also support the hypothesis that the behavior is material and mechanism dependent owing to differences when compared to other materials systems. These 
results have fundamental and practical implications, in that fs-laser-driven pulsed TEM offers a combination of stability and tunability that affords studying specific damage mechanisms and durability of $\mathrm{MAPbI}_{3}$, as well as providing structural, chemical, electronic, and dynamic information from less-damaged specimens.

*Author to whom correspondence should be addressed.

Email: flan0076@umn.edu

Office: +1 612-625-3867

Acknowledgments: This material is based on work supported by the U.S. Department of Energy, Office of Science, Office of Basic Energy Sciences under Award No. DE-SC0018204. This material is based upon work supported by the National Science Foundation Graduate Research Fellowship Program under Grant No. DGE-1348264. RJH acknowledges support from the University of Minnesota Institute on the Environment and Ronald A. and Janet A. Christenson. Part of this work was carried out in the College of Science and Engineering Characterization Facility, University of Minnesota, which has received capital equipment funding from the NSF through the UMN MRSEC program under Award Number DMR2011401.

Author Contributions: EJV contributions were formal analysis, investigation, methodology, software, validation, visualization, writing - original draft, writing - review and editing. CPC contributions were investigation, methodology, specimen preparation, writing - original draft, and writing - review and editing. RJH contributions were conceptualization, funding 
acquisition, project administration, supervision, and writing - review and editing. DJF contributions were conceptualization, formal analysis, funding acquisition, methodology, project administration, resources, supervision, visualization, writing - original draft, writing - review and editing.

Competing Interests: Authors declare no competing interests.

\section{References:}

(1) $\quad$ Egger, D. A.; Bera, A.; Cahen, D.; Hodes, G.; Kirchartz, T.; Kronik, L.; Lovrincic, R.; Rappe, A. M.; Reichman, D. R.; Yaffe, O. What Remains Unexplained about the Properties of Halide Perovskites? Adv. Mater. 2018, 30, 1800691.

(2) Doherty, T. A. S.; Winchester, A. J.; Macpherson, S.; Johnstone, D. N.; Pareek, V.; Tennyson, E. M.; Kosar, S.; Kosasih, F. U.; Anaya, M.; Abdi-Jalebi, M.; AndajiGarmaroudi, Z.; Wong, E. L.; Madéo, J.; Chiang, Y.-H.; Park, J.-S.; Jung, Y.-K.; Petoukhoff, C. E.; Divitini, G.; Man, M. K. L.; Ducati, C.; Walsh, A.; Midgley, P. A.; Dani, K. M.; Stranks, S. D. Performance-Limiting Nanoscale Trap Clusters at Grain Junctions in Halide Perovskites. Nature 2020, 580, 360-366.

(3) Divitini, G.; Cacovich, S.; Matteocci, F.; Cinà, L.; Di Carlo, A.; Ducati, C. In Situ Observation of Heat-Induced Degradation of Perovskite Solar Cells. Nat. Energy 2016, 1, 15012.

(4) Rothmann, M. U.; Li, W.; Zhu, Y.; Liu, A.; Ku, Z.; Bach, U.; Etheridge, J.; Cheng, Y. B. Structural and Chemical Changes to $\mathrm{CH}_{3} \mathrm{NH}_{3} \mathrm{PbI}_{3}$ Induced by Electron and Gallium Ion Beams. Adv. Mater. 2018, 30, 1800629.

(5) Ran, J.; Dyck, O. O.; Wang, X.; Yang, B.; Geohegan, D. B.; Xiao, K. Electron-BeamRelated Studies of Halide Perovskites: Challenges and Opportunities. Adv. Energy Mater. 2020, 10, 1903191.

(6) Ball, J. M.; Petrozza, A. Defects in Perovskite-Halides and Their Effects in Solar Cells. Nat. Energy 2016, 1, 16149.

(7) Rothmann, M. U.; Li, W.; Zhu, Y.; Bach, U.; Spiccia, L.; Etheridge, J.; Cheng, Y.-B. Direct Observation of Intrinsic Twin Domains in Tetragonal $\mathrm{CH}_{3} \mathrm{NH}_{3} \mathrm{PbI}_{3}$. Nat. Commun. 2017, 8, 14547. 
(8) Chen, S.; Zhang, X.; Zhao, J.; Zhang, Y.; Kong, G.; Li, Q.; Li, N.; Yu, Y.; Xu, N.; Zhang, J.; Liu, K.; Zhao, Q.; Cao, J.; Feng, J.; Li, X.; Qi, J.; Yu, D.; Li, J.; Gao, P. Atomic Scale Insights into Structure Instability and Decomposition Pathway of Methylammonium Lead Iodide Perovskite. Nat. Commun. 2018, 9, 4807.

(9) Alberti, A.; Bongiorno, C.; Smecca, E.; Deretzis, I.; Magna, A. L.; Spinella, C. Pb Clustering and $\mathrm{PbI}_{2}$ Nanofragmentation during Methylammonium Lead Iodide Perovskite Degradation. Nat. Commun. 2019, 10, 2196.

(10) Yu, Y.; Zhang, D.; Kisielowski, C.; Dou, L.; Kornienko, N.; Bekenstein, Y.; Wong, A. B.; Alivisatos, A. P.; Yang, P. Atomic Resolution Imaging of Halide Perovskites. Nano Lett. 2016, 16, 7530-7535.

(11) Li, Y.; Zhou, W.; Li, Y.; Huang, W.; Zhang, Z.; Chen, G.; Wang, H.; Wu, G.-H.; Rolston, N.; Vila, R.; Chiu, W.; Cui, Y. Unravelling Degradation Mechanisms and Atomic Structure of Organic-Inorganic Halide Perovskites by Cryo-EM. Joule 2019, 3, 28542866.

(12) Flannigan, D. J.; Lobastov, V. A.; Zewail, A. H. Controlled Nanoscale Mechanical Phenomena Discovered with Ultrafast Electron Microscopy. Angew. Chem. Int. Ed. 2007, 46, 9206-9210.

(13) Kwon, O. H.; Ortalan, V.; Zewail, A. H. Macromolecular Structural Dynamics Visualized by Pulsed Dose Control in 4D Electron Microscopy. Proc. Natl. Acad. Sci. U.S.A. 2011, 108, 6026-6031.

(14) VandenBussche, E. J.; Flannigan, D. J. Reducing Radiation Damage in Soft Matter with Femtosecond-Timed Single-Electron Packets. Nano Lett. 2019, 19, 6687-6694.

(15) Kisielowski, C.; Specht, P.; Freitag, B.; Kieft, E. R.; Verhoeven, W.; van Rens, J. F. M.; Mutsaers, P.; Luiten, J.; Rozeveld, S.; Kang, J.; McKenna, A. J.; Nickias, P.; Yancey, D. F. Discovering Hidden Material Properties of $\mathrm{MgCl}_{2}$ at Atomic Resolution with Structured Temporal Electron Illumination of Picosecond Time Resolution. Adv. Funct. Mater. 2019, 29, 1807818.

(16) Choe, H.; Ponomarev, I.; Montgomery, E.; Lau, J. W.; Zhu, Y.; Zhao, Y.; Liu, A.; Kanareykin, A.; Jing, C. Mitigation of Radiation Damage in Macromolecules via Tunable Ultrafast Stroboscopic TEM. bioRxiv 2020, 2020.2005.2015.099036.

(17) Lang, F.; Nickel, N. H.; Bundesmann, J.; Seidel, S.; Denker, A.; Albrecht, S.; Brus, V. V.; Rappich, J.; Rech, B.; Landi, G.; Neitzert, H. C. Radiation Hardness and Self-Healing of Perovskite Solar Cells. Adv. Mater. 2016, 28, 8726-8731.

(18) Nie, W.; Blancon, J.-C.; Neukirch, A. J.; Appavoo, K.; Tsai, H.; Chhowalla, M.; Alam, M. A.; Sfeir, M. Y.; Katan, C.; Even, J.; Tretiak, S.; Crochet, J. J.; Gupta, G.; Mohite, A. D. Light-Activated Photocurrent Degradation and Self-Healing in Perovskite Solar Cells. Nat. Commun. 2016, 7, 11574. 
(19) Ceratti, D. R.; Rakita, Y.; Cremonesi, L.; Tenne, R.; Kalchenko, V.; Elbaum, M.; Oron, D.; Potenza, M. A. C.; Hodes, G.; Cahen, D. Self-Healing inside $\mathrm{APbBr}_{3} \mathrm{Halide}$ Perovskite Crystals. Adv. Mater. 2018, 30, 1706273.

(20) Egerton, R. F. Radiation Damage to Organic and Inorganic Specimens in the TEM. Micron 2019, 119, 72-87.

(21) Momma, K.; Izumi, F. VESTA 3 for Three-Dimensional Visualization of Crystal, Volumetric and Morphology Data. J. Appl. Crystallogr. 2011, 44, 1272-1276.

(22) Stoumpos, C. C.; Malliakas, C. D.; Kanatzidis, M. G. Semiconducting Tin and Lead Iodide Perovskites with Organic Cations: Phase Transitions, High Mobilities, and NearInfrared Photoluminescent Properties. Inorg. Chem. 2013, 52, 9019-9038.

(23) Yamada, Y.; Yamada, T.; Phuong, L. Q.; Maruyama, N.; Nishimura, H.; Wakamiya, A.; Murata, Y.; Kanemitsu, Y. Dynamic Optical Properties of $\mathrm{CH}_{3} \mathrm{NH}_{3} \mathrm{PbI}_{3}$ Single Crystals As Revealed by One- and Two-Photon Excited Photoluminescence Measurements. J. Am. Chem. Soc. 2015, 137, 10456-10459.

(24) Grubb, D. T. Radiation Damage and Electron Microscopy of Organic Polymers. J. Mater. Sci. 1974, 9, 1715-1736.

(25) Plemmons, D. A.; Flannigan, D. J. Ultrafast Electron Microscopy: Instrument Response from the Single-Electron to High Bunch-Charge Regimes. Chem. Phys. Lett. 2017, 683, 186-192.

(26) Fryer, J. R. Radiation Damage in Organic Crystalline Films. Ultramicroscopy 1984, 14, 227-236.

(27) Pisoni, A.; Jaćimović, J.; Barišić, O. S.; Spina, M.; Gaál, R.; Forró, L.; Horváth, E. UltraLow Thermal Conductivity in Organic-Inorganic Hybrid Perovskite $\mathrm{CH}_{3} \mathrm{NH}_{3} \mathrm{PbI}_{3} . J$. Phys. Chem. Lett. 2014, 5, 2488-2492.

(28) Qian, X.; Gu, X.; Yang, R. Lattice Thermal Conductivity of Organic-Inorganic Hybrid Perovskite $\mathrm{CH}_{3} \mathrm{NH}_{3} \mathrm{PbI}_{3}$. Appl. Phys. Lett. 2016, 108, 063902.

(29) Onoda-Yamamuro, N.; Matsuo, T.; Suga, H. Calorimetric and IR Spectroscopic Studies of Phase Transitions in Methylammonium Trihalogenoplumbates. J. Phys. Chem. Solids 1990, 51, 1383-1395.

(30) Baikie, T.; Barrow, N. S.; Fang, Y.; Keenan, P. J.; Slater, P. R.; Piltz, R. O.; Gutmann, M.; Mhaisalkar, S. G.; White, T. J. A Combined Single Crystal Neutron/X-ray Diffraction and Solid-State Nuclear Magnetic Resonance Study of the Hybrid Perovskites $\mathrm{CH}_{3} \mathrm{NH}_{3} \mathrm{PbX}_{3}$ (X = I, Br and Cl). J. Mater. Chem. A 2015, 3, 9298-9307.

(31) Ghosh, T.; Aharon, S.; Etgar, L.; Ruhman, S. Free Carrier Emergence and Onset of Electron-Phonon Coupling in Methylammonium Lead Halide Perovskite Films. J. Am. Chem. Soc. 2017, 139, 18262-18270. 
Page 16 of 17 


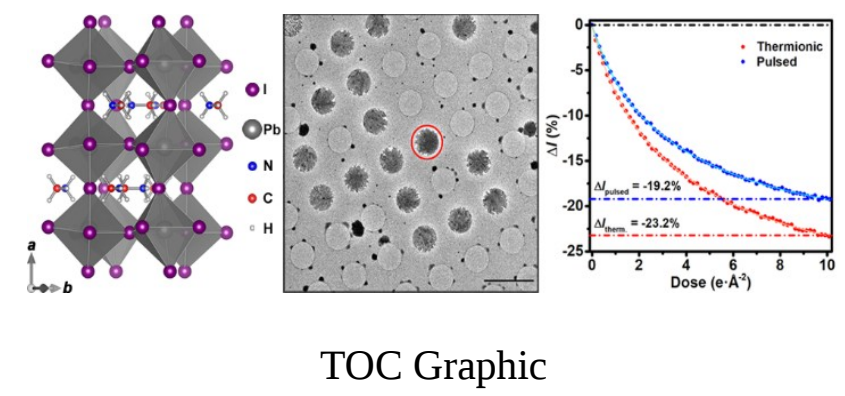

Page 17 of 17 